\title{
A STUDY OF THE NUTRITIONAL DEFECT IN WERNICKE'S SYNDROME
}

\author{
THE EFFECT OF A PURIFIED DIET, THIAMINE, AND OTHER VITAMINS \\ ON THE CLINICAL MANIFESTATIONS ${ }^{1}$
}

\author{
BY GERALD B. PHILLIPS, MAURICE VICTOR, RAYMOND D. ADAMS, AND \\ CHARLES S. DAVIDSON
}

\begin{abstract}
(From the Thorndike Memorial Laboratory, Second and Fourth Medical Services [Harvard], Boston City Hospital, the Department of Neurology, Massachusetts General Hospital and Boston City Hospital, and the Departments of Medicine and Neurology, Harvard Medical School, Boston, Mass.)
\end{abstract}

(Submitted for publication December 15, 1951 ; accepted July 29, 1952)

The salient clinical features of Wernicke's syndrome (1) are partial to complete paralysis of extra-ocular muscles (most commonly the external recti), nystagmus, ataxia and mental disturbances. Postmortem examination reveals changes in the nervous structures adjacent to the third and fourth ventricles and the aqueduct. The lesions are characterized by varying degrees of necrosis of both nerve cells and nerve fibers with appropriate reactions of microglia and astrocytes, alteration of the small blood vessels, and in some cases, petechial hemorrhages (2-5).

The syndrome is usually associated with chronic alcoholism and for that reason has sometimes been attributed to a neurotoxic effect of the alcohol; but the occurrence of the same clinical sequence in non-alcoholic patients with malnutrition $(1,2$, 4-8) has directed attention to nutritional deficiency as the etiological basis. Moreover, similar pathological lesions have been produced in the rat ( 9 , $10)$, fox $(11,12)$, and pigeon $(3,9,13,14)$, by maintaining these animals on thiamine-deficient diets.

The first extensive clinical investigation of the nutritional disorder of Wernicke's syndrome was conducted by Jolliffe, Wortis, and Fein (15), who followed twenty-seven patients on various diets with specific vitamin supplements and concluded that the ophthalmoplegia responded to thiamine but that the whole syndrome was probably due to a "combination of several nutritional deficiencies." Other reports concur that thiamine deficiency

1 The expenses of this investigation were defrayed in part by grants from Merck and Company, Inc., Rahway, New Jersey, and Sharpe and Dohme, Inc., Glenolden, Pennsylvania. plays a definite role in the etiology of this syndrome $(7,8,16)$.

The study reported in the present communication was designed to define more clearly the effect of bed rest, withdrawal of alcohol, and administration of vitamins on the individual clinical signs comprising Wernicke's syndrome. Patients with. typical manifestations of this syndrome were maintained for varying periods of time on a purified diet consisting solely of glucose and minerals. At appropriate times specific vitamins were added. The principal components of the clinical picture, i.e., the ophthalmoplegia, nystagmus, ataxia, and mental disturbances, were examined at frequent intervals.

\section{MATERIALS AND METHODS}

Nine patients with the typical signs of Wernicke's syndrome were observed on the wards of the Boston City Hospital. Only patients with a definite degree of ophthalmoplegia who had received no food after admission were included. All patients were men, white, and over 40 years of age. Dietary histories could not be obtained from eight because of mental disturbances, but it was learned from their families and friends that they were confirmed chronic alcoholics and had been drinking heavily and eating little or no food in the weeks preceding entry to the hospital. Objective evidence of undernutrition, such as looseness of the skin and thinness of muscle, was observed in all cases. In addition, six (Cases Nos. 1 through 6) had liver disease as indicated by abnormal "liver function" tests, and in three (Cases Nos. 1, 3, and 4) this was confirmed by needle biopsy. No evidence of beriberi was found in any patient except for a frank peripheral neuropathy in one (Case No. 6). Pellagrinous skin lesions were seen in only one patient (Case No. 9), whereas two showed tender, red tongues (Cases Nos. 2 and 3). One patient (Case No. 9) had perifollicular hemorrhages and another (Case No. 2) manifested hoarseness. No other signs suggesting deficiency disease were 
detected, and in no case was any other serious abnormality found, except for delirium tremens, which was evident in Case No. 1. Significant fever was not present in any patient. All the patients survived their disease.

The subjects received no food from the time they entered the hospital and all, except Case No. 9, were started immediately on a regimen consisting of the oral administration of a solution which contained $200 \mathrm{gm}$. of glucose and $1.3 \mathrm{gm}$. of sodium chloride per liter of water. Case No. 9 was given an infusion containing multiple vitamins ${ }^{2}$ including thiamine on admission and was started on a regular hospital diet twelve hours later. Cases Nos. 3, 5 , and 6 received potassium chloride in addition to glucose and saline in an attempt to eliminate potassium deficiency as an etiological component of the mental disturbance. Occasionally, prophylactic penicillin and small doses of paraldehyde were given. No other form of sustenance or medication was administered except for certain vitamins as specified below. An exact measure of the amount of the solution consumed by each patient was difficult to obtain, but each ingested approximately 1 to 2 liters a day. After 3 to 11 days of the purified diet, a regular hospital diet was instituted for the remainder of the hospitalization. The period of observation on the purified diet was not extended beyond 11 days because of the serious nature of Wernicke's syndrome. Three subjects vomited infrequently, but it was impossible to determine whether this was due to an alcoholic gastritis, to Wernicke's syndrome, to the possible gastric irritation of glucose and saline, or to some other factor.

All the patients, except Case No. 4, were confined to bed until they were able to walk without assistance. Case No. 6 could not walk during his hospitalization but was allowed to sit in a chair after the third week in the hospital.

The ophthalmoplegia, nystagmus, ataxia, and mental disturbances were observed closely and an attempt was made to quantitate them on a $1+$ to $4+$ scale. Since the principal ocular derangement in all the cases was a bilateral weakness of the external rectus muscles, the power of abduction of the eyes was used as an index of the degree of ophthalmoplegia. A total inability to abduct the eye beyond the midline was considered as $4+$, a slight abductive movement as $3+$, moderate abduction as $2+$ and almost full lateral movement with only a small amount of sclera showing between the limbus and outer canthus or an inability to sustain lateral gaze for more than a few seconds, as $1+$. Coarse, well-sustained nystagmus demonstrated on lateral or vertical gaze was designated as $4+$, lesser degrees of it as $3+$ or $2+$ and a barely perceptible nystagmus as $1+$. With respect to ataxia, $4+$ denoted an inability to walk even with some assistance ; $3+$ meant that the patient could walk if aided;

2 "Berocca C" (Hoffmann-LaRoche). Each 2 cc. contains thiamine hydrochloride, $10 \mathrm{mgm}$; riboflavin, 6 mgm.; niacinamide, $80 \mathrm{mgm}$.; pyridoxine hydrochloride, $6 \mathrm{mgm}$.; panthenol (equivalent to $6 \mathrm{mgm}$. Ca pantothenate), 6 mgm.; ascorbic acid, $100 \mathrm{mgm}$. with $3 \%$ gentisic acid ethanolamide as a preservative.
$2+$ indicated ability to walk alone but with staggering; and $1+$ represented competent locomotion but with slight ataxia. The degree of psychic derangement was most difficult to evaluate. In these patients several aspects of the mental disturbance were discernible, i.e. alertness and the capacity for sustained mental activity, confusion, and memory defect with confabulation. None of the patients exhibited an appreciable variation in the degree of amnesia during the period of observation. Rough quantitative data are given only on the confusional aspect. Four plus implied extreme confusion, often with such total disorientation that the patient did not know whether he was standing or lying, dressed or undressed, indoors or outdoors, or whether it was day or night. He failed to recognize and often misidentified objects and people around him. He could not carry on a conversation or answer questions adequately. Three plus represented the state in which there was greater awareness of the immediate environment, but still gross disorientation as to place and time. Two plus and one plus indicated lesser degrees of confusion, particularly in regard to orientation in place and time. Often confabulation was more evident as the patient improved. None of the patients were disoriented as to person.

\section{RESULTS}

Since the regimen varied somewhat from patient to patient, the clinical data on each will be considered separately.

Case 1 (Table I). This 67-year old man was admitted to the hospital after collapsing in court. His son testified that he was accustomed to drinking in long sprees, the one preceding his admission having lasted 2 months. During this time his diet was said to have consisted of a sandwich at long and irregularly spaced intervals.

He was, on first examination, slightly tremulous and florid. There was considerable loss of muscle. The liver, which was tender and palpable $4 \mathrm{~cm}$. below the right costal margin, showed a large amount of fat and minimal, if any, fibrosis on biopsy; in addition, "liver function" tests were abnormal.

Questions were answered in a normally alert and cooperative manner. $\mathrm{He}$ was disoriented in place and time. In addition, he did not know what clothing he had on; he spoke of lying on a bench in the station waiting to be picked up by his daughter. He did not realize that he was ill and gave quite irrational answers, the responses varying from one minute to the next.

Since he had a coarse tremor and transient hallucinations, he was thought to have delirium tremens in addition to his Wernicke's syndrome. 
TABLE I

Case No. 1-B. R., male, age 67

\begin{tabular}{|c|c|c|c|c|c|c|c|c|c|c|c|c|c|c|c|}
\hline Hospital day & 1 & 2 & 3 & 4 & $5^{*}$ & 6 & 7 & 8* & 9* & 10 & $11^{*}$ & 12 & 15 & 19 & 35 \\
\hline $\begin{array}{l}\text { External } \\
\text { rectus } \\
\text { paralysis }\end{array}$ & $3+$ & $3+$ & $3+$ & $3+$ & $4+$ & $1+$ & \pm & \pm & 0 & 0 & 0 & 0 & 0 & 0 & 0 \\
\hline $\begin{array}{l}\text { Horizontal } \\
\text { nystagmus }\end{array}$ & $3+$ & $3+$ & $3+$ & $3+$ & $3+$ & $3+$ & $3+$ & $2+$ & $2+$ & $1+$ & $1+$ & $1+$ & $1+$ & $1+$ & $1+$ \\
\hline Ataxia & $4+$ & $4+$ & $4+$ & $4+$ & $4+$ & & & $3+$ & $2+$ & $2+$ & $2+$ & $2+$ & & $1+$ & $1+$ \\
\hline Confusion & $4+$ & $4+$ & $3+$ & $3+$ & $3+-$ & $2+$ & $3+$ & $3+$ & $3+$ & $3+$ & $3+$ & $3+$ & $3+$ & $3+$ & $3+$ \\
\hline & Gluc & e and & aline & & $\underset{\substack{100 \\
\text { i.m. }}}{\text { T }}$ & $\begin{array}{r}\text { hiami } \\
2 \\
\end{array}$ & $\begin{array}{l}\text { mgm. } \\
\text { m. } \\
\text { aily }\end{array}$ & & & & \multirow{2}{*}{\multicolumn{5}{|c|}{$\begin{array}{l}\text { Regular } \\
\text { hospital- } \\
\text { diet }\end{array}$}} \\
\hline Therapy & & & & & & & & \begin{tabular}{|c} 
Niacin \\
$1000 \mathrm{mgm}$. \\
p.o. \\
daily \\
\end{tabular} & $\begin{array}{l}\text { Mult } \\
\text { vitan }\end{array}$ & $\begin{array}{l}\text { le } \\
\text { ns } \dagger \rightarrow \rightarrow\end{array}$ & & & & & \\
\hline
\end{tabular}

* Measurements recorded on the day a therapy was started are pre-therapy.

$\dagger$ "Berocca C" (see footnote 2) 4 cc. i.v. daily.

There was a bilateral internal strabismus due to an almost complete external rectus paralysis, as well as a coarse horizontal nystagmus on attempted lateral gaze. His actions betrayed a marked diplopia. Ataxia was manifest on standing; he could not walk even with assistance.

He was given only glucose and saline for four days, during which time his external rectus paralysis became even worse, the nystagmus and ataxia remained the same, his hallucinations and tremor disappeared, and he seemed somewhat less confused. On the fifth day he was started on 100 mgm. of thiamine intramuscularly twice a day in addition to the glucose and saline. Within 5 hours after the first dose, his external rectus paralysis was barely detectable. After three days of thiamine (Day 8), external rectus weakness was only questionably present, nystagmus was less, and walking was accomplished with assistance, but his mental state, which seemed to have temporarily improved within 24 hours after thiamine was instituted, was no better than before thiamine. On Day 8, $1000 \mathrm{mgm}$. of niacin orally per day was added. On Day 9, no external rectus weakness could be detected, and he walked without assistance for the first time; the mental status, however, showed no change. On this day, a multiple vita- min preparation, ${ }^{3}$ given intravenously, was added to the regimen. On Day 10, nystagmus was slightly less and ataxia and mental status were the same as on the previous day. Food was started on Day 11. Thirty-five days after entry, the patient had no ocular palsies, minimal horizontal nystagmus, and a somewhat wide-based gait. His mental disorganization was as marked as prior to the institution of thiamine. Confabulation was more spontaneous at this time than on entry.

Case 2 (Table II). This 57-year old single man was discovered in his room in an irrational and neglected state. He had been there for an indeterminate period of time and was quite unable to supply a history of his illness.

He was very thin and unkempt. His liver was enlarged to two finger-breadths below the right costal margin, and there was laboratory evidence of abnormal function. He was alert, but inattentive and incapable of sustaining a conversation. His remarks and behavior were facetious and inappropriate. He had no idea of the place or date and could give no rational answers to any questions concerning his past history or circumstances of his arrival to the hospital. There was diplopia in all directions, and he usually kept one eye closed.

\footnotetext{
3 "Berocca C"; 4 cc. were given daily.
} 
TABLE II

Case No. 2-E. H., male, age 57

\begin{tabular}{|c|c|c|c|c|c|c|c|c|c|c|c|}
\hline Hospital day & 1 & 2 & 3 & 4 & $5^{*}$ & 6 & 7 & $8^{*}$ & 9 & $10^{*}$ & 18 \\
\hline $\begin{array}{c}\text { External rectus } \\
\text { paralysis }\end{array}$ & $3+$ & $3+$ & $4+$ & $4+$ & $4+$ & $1+$ & $1+$ & 0 & 0 & & 0 \\
\hline $\begin{array}{l}\text { Horizontal } \\
\text { nystagmus }\end{array}$ & 0 & 0 & 0 & 0 & 0 & $3+$ & $3+$ & $2+$ & $1+$ & & $1+$ \\
\hline Ataxia & $3+$ & $3+$ & $3+$ & $3+$ & $3+$ & $2+$ & $2+$ & $1+$ & $1+$ & & $1+$ \\
\hline Confusion & $3+$ & $3+$ & $3+$ & $3+$ & $3+$ & $3+$ & $3+$ & $3+$ & $3+$ & $3+$ & $3+$ \\
\hline \multirow[t]{2}{*}{ Therapy } & Gluco & and sa & e-- & 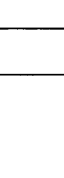 & $\begin{array}{c}\text { Thiar } \\
200 \text { mgm. } \\
\text { i.m. }\end{array}$ & $\begin{array}{l}\text { mine } \\
200 \text { mgm. } \\
\text { s.c. } \\
\text { daily }\end{array}$ & & \multirow{2}{*}{\multicolumn{2}{|c|}{$\underset{\text { vitamins } \dagger}{\text { Multiple }} \longrightarrow$}} & \multirow{2}{*}{\multicolumn{2}{|c|}{$\begin{array}{l}\text { Regular } \\
\text { hospital- } \\
\text { diet }\end{array}$}} \\
\hline & & & & & & & & & & & \\
\hline
\end{tabular}

* See footnote to Table I.

† "Berocca C" (see footnote 2) 4 cc. i.m. daily.

Abduction of each eye was limited to a few millimeters, and nystagmus was not evident. $\mathrm{He}$ needed considerable coaxing and support to stand or take a few steps, both of which acts were carried out in a markedly ataxic manner. Ankle jerks were absent and knee jerks depressed, but there were no other signs of neuropathy.

After four days of the glucose and saline regimen, the paralysis of his external recti was complete. Nystagmus was absent and the ataxia remained unchanged. His responses were even less adequate than on entry and he was more careless of his speech and dress. One hundred mgm. of thiamine were then injected intramuscularly and four hours later, there having been no improvement, another $100 \mathrm{mgm}$. were given. Within a half hour after the second dose, strabismus and diplopia had disappeared, external rectus motion was almost normal, and a coarse horizontal nystagmus became evident for the first time. After three days of thiamine administration, external rectus paralysis was gone, nystagmus was less and ataxia was much improved; his confusional state, however, remained unchanged. A multiple vitamin preparation $^{3}$ was given intramuscularly for the next two days without discernible improvement in the mental state. On Day 10 food was started. Eighteen days after admission, his ocular disturbance was limited to a fine horizontal nystagmus on far lateral gaze, and his gait was slightly wide-based and unsteady. He was more alert, entered into sustained conversation more readily, and required no stimulation to confabulate. His confusion, however, was as marked as prior to the administration of thiamine and other vitamins.

Case 3 (Table III). This 46-year old man, who had been hospitalized nine months previously for cirrhosis of the liver, was readmitted because of irrational behavior and inability to walk for three to four days.

Since youth he had been consuming on the average of 2 qts. of wine daily. His family stated that for at least three years he had eaten only one meal daily, and then "only picked at his food."

There was general emaciation, roughness and dryness of the skin, and enlargement of the liver to $10 \mathrm{~cm}$. below the right costal margin. "Liver function" tests were deranged, and a liver biopsy revealed a large amount of fat with definite fibrosis.

The patient was alert and not tremulous or hallucinated. He realized he was in bed, but literally nothing more. His conversation showed a total lack of understanding of what was going on around him and he misidentified the examiner and other patients. Disorientation in place and time was severe. In addition, there was a pronounced memory defect from the time of entry, and the tendency to confabulate became obvious within a few days. 
TABLE III

Case No. 3-P. F., male, age 46

\begin{tabular}{l|l|l|l|l|l|l|l|l|l|l}
\hline \hline \multicolumn{1}{c|}{ Hospital day } & 1 & 2 & $3^{*}$ & 4 & 5 & 6 & 7 & 8 & $9 *$ & 20 \\
\hline $\begin{array}{l}\text { External rectus } \\
\text { paralysis }\end{array}$ & $2+$ & $2+$ & $4+\dagger$ & \pm & \pm & 0 & 0 & 0 & 0 & 0 \\
\hline Horizontal nystagmus & $1+$ & $1+$ & 0 & $1+$ & $1+$ & $1+$ & $1+$ & $1+$ & 0 & 0 \\
\hline $\begin{array}{l}\text { Vertical nystagmus } \\
\text { Ataxia }\end{array}$ & 0 & 0 & 0 & $1+$ & $1+$ & 0 & 0 & 0 & 0 & 0 \\
\hline Confusion & $3+$ & $3+$ & $3+$ & $2+$ & $2+$ & $2+$ & $2+$ & $2+$ & $1+$ & $1+$ \\
\hline & $3+$ & $3+$ & $3+$ & $3+$ & $3+$ & $3+$ & $3+$ & $3+$ & $3+$ & $3+$ \\
\hline
\end{tabular}

* See footnote to Table I.

$\uparrow$ Paralysis of lateral gaze.

On entry there was a mild internal strabismus; abduction of both eyes was incomplete and a mild horizontal nystagmus developed on attempting this movement. The pupils were normal in size and reacted briskly to light. His gait was ataxic, and he required support to walk. Knee and ankle jerks were absent, but motor power and sensation were adequate.

On the third day of the glucose and saline regimen, there was an increase of the ocular defect manifested by a paralysis of gaze; there was almost a complete inability to perform lateral conjugate movements of the eyes although upward and downward gaze was unimpaired. Moreover, the pupils became miotic and non-reacting. Five milligrams of thiamine were then administered intravenously. Within six hours, lateral gaze was almost normal, and the pupils became dilated and reactive to light. After five subsequent days of thiamine (15 mgm. orally per day) in addition to the glucose and saline, external rectus paralysis and nystagmus were not detectable, and the ataxia was only slight in degree. The mental disturbance, however, was unimproved. After 11 subsequent days of food, ataxia was evident only on heel-totoe walking, and there was still no change in mental status.

Case 4 (Table IV). This 43-year old man was admitted because of advanced liver disease and complaints of double vision. He gave a history of alcoholic excess ( 50 to 60 glasses of ale daily) for 20 years. In the last three months anorexia was extreme, and he claimed to have lost 25 pounds in weight in the month preceding entry into the hospital. Diplopia was present for seven days before admission. On physical examination, his liver was found to be enlarged to three to four finger-breadths below the right costal margin; "liver function" tests were markedly altered and a liver biopsy revealed a marked degree of fat and fibrosis.

When first examined, no mental defect was demonstrable. The abnormal eye signs consisted of an alternating internal strabismus, a moderate bilateral external rectus weakness, and a coarse horizontal nystagmus on lateral gaze. Ataxia was slight and difficult to assess because of an old right peroneal palsy.

On the second day of the glucose and saline diet he developed a quiet confusion, which increased over the subsequent two days to the point where he was wandering aimlessly around the ward, disoriented, irrational, and unaware of the meaning of all that was happening around him. The ocular disorder and ataxia remained unchanged. On Day 4 he was given $500 \mathrm{mgm}$. of niacin orally; 24 hours later (Day 5) his condition was no different except for an increase in the external rectus weakness and a decrease in horizontal nystagmus. On. Day 5 he was given 50 mgm. of thiamine intravenously and in one and a quarter hours there was a definite decrease in external rectus palsy; in one 
TABLE IV

Case No. 4-P. S., Male, age 43

\begin{tabular}{l|c|c|c|c|c|c|c|c|c|c|c|c}
\hline \multicolumn{1}{c|}{ Hospital day } & 1 & 2 & 3 & $4^{*}$ & $5^{*}$ & $6^{*}$ & 7 & 8 & 9 & 10 & 11 & 14 \\
\hline $\begin{array}{l}\text { External rectus } \\
\text { paralysis }\end{array}$ & $2+$ & $2+$ & $2+$ & $2+$ & $3+$ & 0 & 0 & 0 & 0 & 0 & 0 & 0 \\
\hline Horizontal nystagmus & $3+$ & $3+$ & $3+$ & $3+$ & $2+$ & $2+$ & $2+$ & $1+$ & & & $1+$ & \pm \\
\hline Ataxia & $1+$ & $1+$ & $1+$ & $1+$ & $1+$ & $1+$ & $1+$ & $1+$ & & & \\
\hline Confusion & 0 & $1+$ & $2+$ & $3+$ & $3+$ & $3+$ & 0 & $2+$ & $2+$ & 0 & 0 \\
\hline Therapy & Glucose and saline & $\begin{array}{c}\text { Niacin } \\
\text { p.o. }\end{array}$ & $\begin{array}{c}\text { Thiamine } \\
50 \text { mgm. } \\
\text { i.v. } \\
\text { Niacin } \\
1000 \text { mgm. } \\
\text { p.o. } \\
\text { Multiple } \\
\text { vitamins }\end{array}$ & $\begin{array}{l}\text { Regular } \\
\text { hospital } \\
\text { diet }\end{array}$ \\
\hline
\end{tabular}

* See footnote to Table I.

† See footnote to Table I.

and three-quarter hours he could abduct his eyes almost to their full extent, and in three hours he had full range of movement, but still had difficulty in sustaining lateral gaze. Several hours later he was given $1000 \mathrm{mgm}$. of niacin orally and a multiple vitamin preparation ${ }^{3}$ intravenously. On Day 6,48 hours after starting niacin and 24 hours after thiamine and multiple vitamins, he was still thoroughly confused even though no external rectus weakness was evident and nystagmus was less.
The regular hospital diet was begun that evening and when seen the next morning ( 12 hours later) he showed no mental abnormality, but still had nystagmus and slight ataxia. One day later, however, he lapsed into a confused state, which reversed completely within 36 hours. On the fourteenth day after admission he had only a questionable nystagmus, but no abnormality of ocular movement, gait, or mental function.

Case 5 (Table $V)$. This 58-year old man

TABLE V

Case No. 5-C. Z., male, age 58

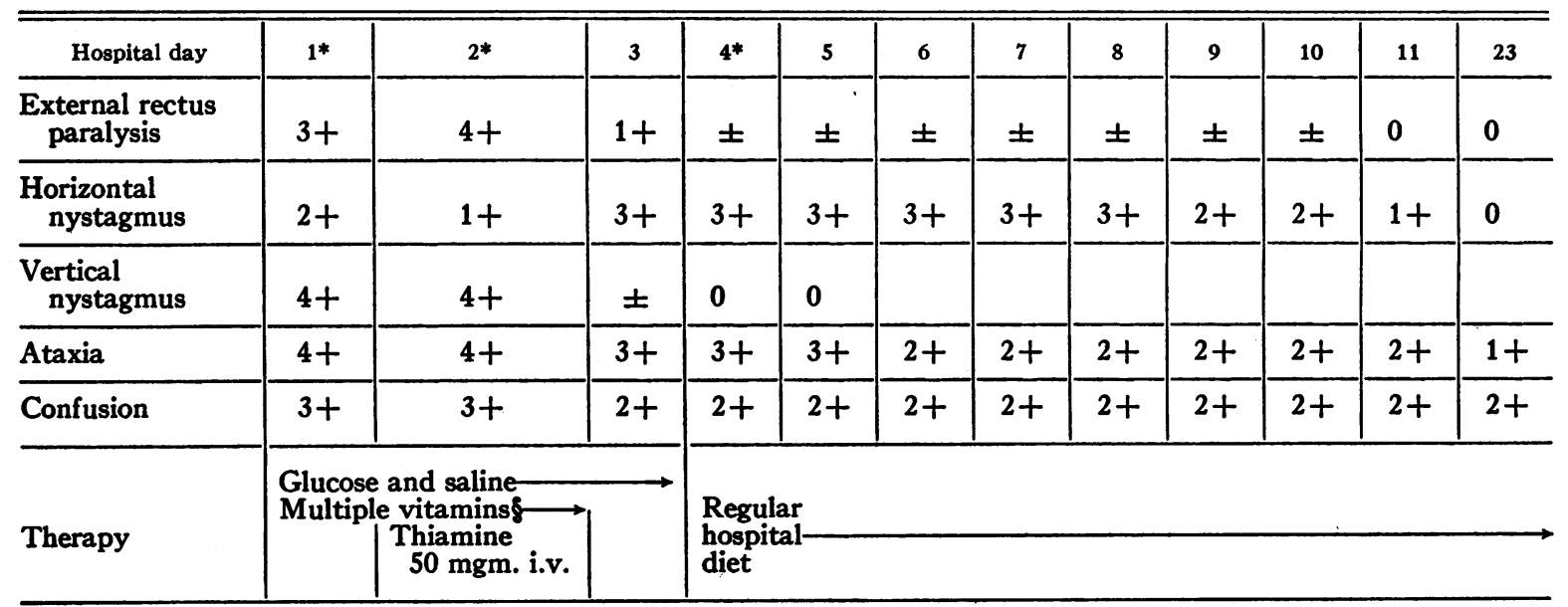

* See footnote to Table I.

8 The following vitamins were given orally daily: $\mathrm{Ca}$ pantothenate, 100 mgm.; pyridoxine, $100 \mathrm{mgm}$.; folic acid, $10 \mathrm{mgm}$.; ascorbic acid, $250 \mathrm{mgm}$.; riboflavin, $10 \mathrm{mgm}$.; niacin, $250 \mathrm{mgm}$. In addition, $10 \mu \mathrm{gm}$. of vitamin $\mathrm{B}_{12} \mathrm{were}$ administered intramuscularly on Day 1. 
was brought to the hospital by the police because of the acute onset of confusion and abdominal distress. He had been leading a derelict's existence for many years, moving from one job and lodging house to another and drinking steadily. In the months preceding his admission, his family had noted an aversion to food and a severe weight loss of undetermined amount.

$\mathrm{He}$ was a wasted, untidy man with evidence of mild liver disease. He was alert and cheerful and not hallucinating. Orientation in place and time, recent and past memory, judgment and insight were all impaired. His ocular defect consisted of internal strabismus, almost complete paralysis of the external recti, and a marked vertical and fine horizontal nystagmus. He exhibited severe ataxia, despite the presence of normal reflexes, motor power and sensation.

He was given glucose and saline and multiple vitamins (see Table V). Twenty-four hours later his 'condition was the same except that his external rectus paralysis had become complete and horizontal nystagmus less. The vitamins of the previous day were repeated, except for vitamin $B_{12}$, with no further change. Later in the day he was given $50 \mathrm{mgm}$. of thiamine intravenously and within four hours he could abduct his eyes almost completely. Associated with this improvement was an increase in horizontal nystagmus. On Day 3 , only slight external rectus weakness was evident and although the horizontal nystagmus was still rather marked, the presence of any vertical nystagmus was doubtful. Ataxia was somewhat less and his mental status was improved to the extent that he was now oriented in place; also, confabulation was now becoming prominent.

On Day 4, food was started, and 19 days later no ocular abnormality of any kind was elicitable, and ataxia was slight in degree. He was oriented in place but grossly disoriented in time; no improvement in retentive memory had occurred; but confabulation was more easily provoked than on admission.

Case 6 (Table VI). This 50-year old man was admitted because of irrationality and inability to walk of two days duration. On physical examination, his liver was found to be three finger-breadths below the right costal margin. In addition, there were slight abnormalities of the "liver function" tests.

On entry he was profoundly confused in place and time as well as to his immediate bodily environment. He had no idea what clothing he had on or who the examiner was. His memory was equally badly disordered, and confabulation was detectable at the first examination. He showed a moderate external rectus weakness, slight horizontal nystagmus and diplopia. Standing was impossible because of a severe peripheral neuropathy, and thus ataxia could not be assessed.

During the first four days of glucose and saline his nystagmus and confusion grew worse, and

TABLE VI

Case No. 6\|l-J. M., male, age 50

\begin{tabular}{|c|c|c|c|c|c|c|c|c|c|c|c|c|}
\hline Hospital day & 1 & 2 & 3 & 4 & $5^{*}$ & 6 & $7 *$ & 8 & 9 & 10 & 23 & 35 \\
\hline $\begin{array}{l}\text { External rectus } \\
\text { paralysis }\end{array}$ & $2+$ & $2+$ & $2+$ & $2+$ & $2+$ & $3+$ & $4+\ddagger$ & $1+$ & $1+$ & $1+$ & 0 & 0 \\
\hline $\begin{array}{l}\text { Horizontal } \\
\text { nystagmus }\end{array}$ & $1+$ & $1+$ & $1+$ & $2+$ & $2+$ & 0 & 0 & $3+$ & $3+$ & & $3+$ & $3+$ \\
\hline Confusion & $3+$ & $3+$ & $3+$ & $4+$ & $4+$ & $4+$ & $4+$ & $3+$ & $3+$ & $3+$ & $3+$ & $3+$ \\
\hline Therapy & Gluco & and s & ne- & & \multicolumn{2}{|c|}{$\begin{array}{l}\text { Multiple } \\
\text { vitamins } \delta\end{array}$} & $\begin{array}{c}\text { Thiamine } \\
100 \mathrm{mgm} \text {. } \\
\text { i.v. }\end{array}$ & \multicolumn{5}{|c|}{$\begin{array}{l}\text { Regular } \\
\text { hospital- } \\
\text { diet }\end{array}$} \\
\hline
\end{tabular}

* See footnote to Table I.

$\ddagger$ Paralysis of gaze.

The following vitamins were given orally daily: Ca pantothenate, $100 \mathrm{mgm}$.; pyridoxine, $100 \mathrm{mgm}$.; folic acid, $10 \mathrm{mgm}$.; ascorbic acid, $500 \mathrm{mgm}$.; riboflavin, $10 \mathrm{mgm}$.; niacin, $500 \mathrm{mgm}$. In addition, $10 \mu \mathrm{gm}$. of vitamin $\mathrm{B}_{12} \mathrm{were}$ administered intramuscularly on Day 5.

II No evaluation of ataxia was possible because of peripheral neuropathy.

T Started evening of Day 7. 
TABLE VII

Case No. 7-F. L., male, age 60

\begin{tabular}{l|l|l|l|l|l|l|l|l|l|l|l|l}
\hline \multicolumn{1}{c|}{ Hospital doy } & $1^{*}$ & 2 & 3 & 4 & 5 & 6 & 10 & $11^{*}$ & 12 & 15 & 18 & 29 \\
\hline $\begin{array}{l}\text { External rectus } \\
\text { paralysis }\end{array}$ & $3+$ & $1+$ & $1+$ & $1+$ & $1+$ & $1+$ & $1+$ & 0 & 0 & 0 & 0 & 0 \\
\hline Horizontal nystagmus & 0 & $3+$ & $3+$ & $3+$ & $3+$ & $3+$ & $3+$ & $3+$ & $3+$ & $3+$ & $3+$ & $3+$ \\
\hline \begin{tabular}{l} 
Vertical nystagmus \\
\hline Ataxia
\end{tabular} & $2+$ & $2+$ & $2+$ & $2+$ & $2+$ & 0 & 0 & 0 & 0 & 0 & 0 & 0 \\
\hline Confusion & $4+$ & - & - & - & $3+$ & $2+$ & $2+$ & $2+$ & $2+$ & $1+$ & $1+$ & $1+$ \\
\hline Therapy & $3+$ & $3+$ & $3+$ & $3+$ & $3+$ & $3+$ & $3+$ & $3+$ & $3+$ & $3+$ & $3+$ & $3+$ \\
\hline
\end{tabular}

* See footnote to Table I.

there was an increase in general weakness. On Day 5, he was given several vitamins exclusive of thiamine (see Table VI). On Day 6, there was almost complete external rectus paralysis with a concomitant disappearance of nystagmus. He was noisy, irrational, uncooperative and generally weaker. By Day 7, he had lost all movements of his eyes except for upward gaze, and a vertical nystagmus was noted for the first time. One hundred milligrams of thiamine were then injected intravenously and within 2 hours lateral conjugate movements improved considerably and horizontal nystagmus became prominent. A few hours later food was started. On Day 8, his external rectus weakness was slight and he was generally more alert and cooperative and, perhaps, less confused. Thirty-five days after admission, examination disclosed absence of external rectus weakness, a fine vertical nystagmus, and a coarse horizontal nystagmus. His mental status, except for increased alertness and attentiveness, was unchanged. The neuropathy was still very severe.

Case 7 (Table VII). This 60-year old man collapsed in court while answering charges of drunkenness. He showed generalized wasting but no definite evidence of liver disease. He lay quietly in bed and would reply only to persistent questioning, being quite content to turn over and suspend the conversation. He could give only his name correctly. He thought he was in a schoolhouse, mistook the examiner for a saleswoman, and had no idea of the date, his address, or the meaning of what he saw around him. His eyes could be abducted only slightly from the mid-line, and no horizontal nystagmus was demonstrable. Upward gaze was limited, and a vertical nystagmus developed on this movement. $\mathrm{He}$ was unable to stand without help and reeled when attempting to walk. Motor power was intact, but knee and ankle jerks could not be obtained.

After the initial examination, the diet of glucose and saline was instituted and continued for ten days. He was given, on entry and once daily thereafter, $100 \mathrm{mgm}$. of thiamine intramuscularly as the only supplement.

Three hours after the first injection of thiamine, ocular movement was improved, and a coarse horizontal nystagmus appeared; 12 hours later abduction was almost complete. Over the next nine days there was gradual improvement in his ability to sustain lateral gaze. Vertical nystagmus disappeared by the sixth day, but the horizontal nystagmus remained unchanged from the time it appeared. The ataxia showed slow but progressive improvement over the ten-day period; at the end of this time he was getting about freely, although his gait was still wide-based, and he staggered slightly on turning quickly. Mentally the patient became more alert and cheerful. He entered into conversation readily and confabulated freely. Only in these respects was there a change in his mental status since admission. Food was started on the eleventh day. Eighteen days later ataxia was further improved but no essential change could be detected in his horizontal nystagmus or confusion. 
Case 8 (Table VIII). This 74-year old man was brought to the hospital by the police after having collapsed in a doorway. He was poorly nourished and his skin was dry, loose, and covered with pediculous excoriations. No definite evidence of liver disease was detected. He spent most of the day lying in bed with his eyes closed, a state from which he could be aroused easily. He then answered questions alertly, although he did not enter into spontaneous conversation. $\mathrm{He}$ was consistently and grossly disoriented in time, being wrong as to the season, and giving the year as 1970. His confusion in regard to his surroundings varied. At times he thought he was in a hospital, at others in a hotel. Memory span was impaired to the extent that he could not recall two facts after a lapse of one minute. Confabulation could be elicited at the first examination. There was marked bilateral external rectus weakness, and a coarse horizontal nystagmus developed on lateral gaze. The patient walked very slowly, with small uncertain steps, requiring support occasionally, especially on turning. There were no signs of peripheral neuropathy.

After an observation period of 24 hours, the glucose and saline diet was instituted and continued for 11 days, with a daily supplement of $100 \mathrm{mgm}$. of thiamine intramuscularly for the first ten days. The patient was not seen again until 12 hours after the first dose of thiamine, at which time there was a marked improvement in the external rectus palsy; complete abduction, however, was accomplished only after five days of therapy. The ny- stagmus also was reduced in degree within 12 hours; after three days it was barely detectable on left lateral gaze only, a state which then persisted for as long as the patient was followed. His ataxia improved progressively over the ten days, although it was still evident at the end of this time.

By the end of the ten-day period of the purified diet and thiamine administration, there was a slight but definite improvement in mental state. He was less confused in time, consistently giving the correct month and approximating the year. When questioned on general information as well as events in his own life, he gave many more correct answers than on admission. His memory span was increased by several minutes; confabulation was unchanged.

A multiple vitamin preparation ${ }^{3}$ was given intramuscularly on Day 12 with no further change in the patient's condition. One day later food was started and after four days of the regular hospital diet, there was still no discernible change.

Case 9. This 68-year old man was admitted after collapsing in the street. He showed signs of general neglect and marked wasting. There were numerous petechiae over his thighs and knees suggesting scurvy. No evidence of liver disease was found. He was disinclined to speak, but his few responses indicated profound disorientation and memory loss. His eyes showed an almost complete external rectus paralysis, coarse horizontal nystagmus, and fine vertical nystagmus. His gait was broad-based and his steps short and uncertain

TABLE VIII

Case No. 8-D. S., male, age 74

\begin{tabular}{l|c|c|c|c|c|c|c|c|c|c|c|c|c|c}
\hline \hline Hospital day & 1 & $2 *$ & 3 & 4 & 5 & 6 & 7 & 8 & 9 & 10 & 11 & $12^{*}$ & $13^{*}$ & 17 \\
\hline $\begin{array}{c}\text { External rectus } \\
\text { paralysis }\end{array}$ & $3+$ & $3+$ & $1+$ & $1+$ & $1+$ & $1+$ & 0 & 0 & 0 & 0 & 0 & 0 & 0 & 0 \\
\hline $\begin{array}{l}\text { Horizontal } \\
\text { nystagmus }\end{array}$ & $3+$ & $3+$ & $2+$ & $1+$ & $1+$ & $1+$ & $1+$ & $1+$ & $1+$ & $1+$ & $1+$ & $1+$ & $1+$ & $1+$ \\
\hline Ataxia & $3+$ & $3+$ & $3+$ & $2+$ & $1+$ & $1+$ & $1+$ & $1+$ & $1+$ & $1+$ & $1+$ & $1+$ & $1+$ & $1+$ \\
\hline Confusion & $3+$ & $3+$ & $3+$ & $3+$ & $3+$ & $3+$ & $3+$ & $2+$ & $2+$ & $2+$ & $2+$ & $2+$ & $2+$ & $2+$ \\
\hline Therapy & $\begin{array}{l}\text { Glucose and saline- } \\
\text { Thiamine } \\
\text { 100 mgm. } \\
\text { i.m. daily }\end{array}$ \\
\hline
\end{tabular}

* See footnote to Table I.

t See footnote to Table II. 
in the face of adequate motor power, normal reflexes, and intact sensory function.

On the night of admission he was given 500 mgm. of thiamine and a multiple vitamin preparation ${ }^{4}$ intravenously. When he was next seen twelve hours later, his external rectus weakness was strikingly improved and consisted only of an inability to sustain lateral gaze. Otherwise, his condition was essentially unchanged. Food was started at this time. Four days later no external rectus weakness was detectable, but nystagmus and ataxia were the same; the patient was much more alert and cheerful, and he could be induced to confabulate. Re-evaluation 40 days after admission disclosed no oculomotor weakness and only slight horizontal and vertical nystagmus. Ataxia was present only on attempting to walk a straight line. Memory for recent events was virtually nil, and he confabulated freely. In other respects his mental derangement was much the same as it had been four days after admission.

\section{DISCUSSION}

The patients included in this study were selected on the basis of ophthalmoplegia, but it is noteworthy that all of them exhibited some degree of nystagmus, ataxia and mental disturbance. This combination of clinical findings is so distinctive that there can be little doubt as to the accuracy of the diagnosis.

The ophthalmoplegia was surprisingly uniform. In all the patients, it consisted on admission of bilateral external rectus muscle weakness, which was usually asymmetrical in degree. Of the six patients who were sustained on glucose and saline before the institution of thiamine, the ocular paralysis became worse in all, progressing to an almost complete paralysis of gaze in two (Cases Nos. 3 and 6). In another of these patients (Case No. 4) a definite increase in the degree of ocular paralysis occurred within 24 hours after niacin alone was added to the purified diet, and in two (Cases Nos. 5 and 6) within 24 hours after the addition of multiple vitamins exclusive of thiamine. The possibility of an accentuation of a thiamine depletion by the addition of other vitamins deserves consideration in these three patients, especially in view of the experiments of Alexander, Pijoan, and

\footnotetext{
4 "Berocca C."
}

Myerson (13), who claimed that the characteristic changes of Wernicke's encephalopathy could be produced in pigeons more easily if large supplies of other vitamins were added.

The most striking response to thiamine therapy in these patients was the rapid improvement in the ocular palsies, which cleared considerably within one and a quarter to six hours following parenteral administration of thiamine (after as little as $5 \mathbf{~ m g m}$. of this vitamin given to one patient). Nevertheless, it sometimes took several days for the residual weakness to disappear entirely. The miotic, nonreacting, pupils of one patient (Case No. 3), which developed while on glucose and saline only, returned to normal as the external ocular palsies cleared.

The rapid reversal of the ophthalmoplegia with thiamine makes it unlikely that these paralyses resulted from the structural lesion which is considered to be so characteristic of Wernicke's syndrome. Instead, it implies that this part of the clinical disorder was biochemical in nature. The pathological study of Riggs and Boles (4) lends support to this interpretation, for in five cases with oculomotor paralysis the ocular nuclei were not significantly damaged. Furthermore, Prickett (9) in a study of thiamine deficient rats and pigeons also noted a prompt reversal of the neurological disturbances after the administration of thiamine. Such dramatic response to thiamine, moreover, leaves little doubt as to the specificity of this vitamin for the ophthalmoplegia of Wernicke's syndrome. An important clinical lesson to be obtained from this material is that the ophthalmoplegia, which is an essential diagnostic criterion of Wernicke's syndrome, may be eradicated promptly by the administration of thiamine, either parenterally or possibly in the diet. Many of these cases are not then recognized as having Wernicke's syndrome.

Some degree of horizontal nystagmus occurred in all of the patients. Only in recent years has the significance of nystagmus been fully appreciated. de Wardener and Lennox (7), who studied 52 patients with Wernicke's syndrome, described nystagmus as the earliest ocular sign. In our patients during the glucose and saline control period, the horizontal nystagmus decreased only in association with an increase in ocular palsy and returned in full force after the ocular palsy was re- 
lieved by thiamine, indicating that it did not actually improve in the pre-thiamine period but had become less apparent because of increased paralysis of eye muscles. Improvement in nystagmus after thiamine, unlike ocular weakness, tended to be more gradual. Two patients (Cases Nos. 1 and 2), whose horizontal nystagmus did not improve over a four-day control period, showed definite decrease within three days after thiamine alone was added; in another (Case No. 8) there was marked improvement within one day of addition of thiamine alone. In one patient (Case No. 5) the marked vertical nystagmus present on admission showed no improvement after 24 hours on a group of vitamins exclusive of thiamine but cleared almost completely within 12 hours after thiamine only was added. In another (Case No. 7) the vertical nystagmus disappeared within five days after the institution of thiamine alone. These results suggest that the horizontal and vertical nystagmus also respond to thiamine. In four patients (Cases Nos. 1, 6, 7, and 9), however, nystagmus was still present after a month in the hospital. Two of these (Cases Nos. 6 and 7) failed to show any decrease in nystagmus after 27 and 18 days on food, respectively. Indeed, nystagmus has been noted to persist for months or years $(17,8)$ after an attack of Wernicke's syndrome.

Seven patients in this group had a markedly ataxic gait on admission, one (Case No. 4) showed only slight ataxia, and one (Case No. 6) could not be tested for character of gait because of the presence of a polyneuropathy which was so severe that he could not stand. The ataxia was essentially one of stance and gait and could seldom be well demonstrated in movements of the arms and legs individually, such as by finger-to-nose and heel-toshin testing. Like the nystagmus, the ataxia was slow to improve, and of the seven patients who demonstrated it to a marked degree on admission, only one recovered from his ataxia completely, while six still showed it to a slight but definite degree when last examined. In no patient was there any improvement during the pre-thiamine control period, but in two (Cases Nos. 1 and 2), following a four-day control period, there was definite improvement within three days and one day, respectively, after thiamine alone was added. Similarly, Case No. 3, following a two-day control period, showed decrease in ataxia one day after thiamine was started and continued to improve on glucose and saline and thiamine only. In Cases Nos. 7 and 8 , where glucose and saline and thiamine alone were given over a ten-day period, there was progressive improvement in the ataxic gait. It is noteworthy that in these five patients, the ataxia improved despite a low caloric intake. The ataxia, therefore, also seems to be related to a deficiency of thiamine, but because of the slow responses conclusions on this point are less certain than in the case of the ophthalmoplegia.

The mental component of the Wernicke syndrome presented the greatest difficulty in evaluation. Of the nine patients, only one (Case No. 4) was lucid on admission; however, he became increasingly confused during the period of glucose and saline administration and showed no improvement 48 hours after niacin and 24 hours after thiamine was started. Food was then given and in 12 hours he was again mentally clear; a day later, however, he developed an episode of confusion, which lasted 36 hours. It was impossible in this instance to determine whether improvement was due to a delayed effect of niacin or thiamine, to some other substance in food, to a spontaneous recovery from an unrelated confusional psychosis such as delirium tremens, or to some other factor.

The remaining eight patients all showed a profound degree of mental disorder on admission to the hospital. With only minor variations, certain features were common to all. When first seen the patients were alert, although not hyperactive or hallucinating. Often, their attitude was one of complete disinterest, rather than drowsiness. Spontaneous speech was minimal; they. tended to answer questions in a perfunctory manner, with no desire to sustain a conversation. They were unable to focus their attention on any one topic, and would often suspend a conversation. The questions that were answered betrayed disorientation in time and place, misidentification of the people attending them, and complete inability to grasp the meaning of their illness or what was going on around them. In addition, many of their remarks were irrational, nor did these show any consistency from one moment to the next. If the examiner was persistent in his questioning, it was also obvious that retentive memory, especially for recent events, was already an important feature of the general mental disorganization. 
The eventual outcome of the mental disturbance varied to some degree. One patient (Case No. 5) seemed to clear somewhat within 48 hours after receiving multiple vitamins and 12 hours after thiamine, and another (Case No. 8) became slightly less confused while maintained on glucose and saline and thiamine only. The improvement in these two patients was mainly in their orientation in place. In the other six patients, no change of this sort could be detected. If one measured the difference in mental state in terms of increased alertness, cheerfulness, ability to concentrate and sustain a conversation, and ready confabulation, then some degree of change occurred in most of the patients. Since this change occurred only after thiamine was added to the diet, it is possible that this aspect of the mental disorder was related to thiamine deficiency. With respect to memory defect and confabulation and to a lesser extent confusion, no significant improvement could be discerned in these patients within the periods of observation. Since these aspects of the mental disturbances characteristically resolve slowly, if at all (17), the periods of observation on the purified diet may have been too short to evaluate the effect of thiamine. This failure to improve after thiamine administration, therefore, does not exclude thiamine deficiency as a cause of the mental disturbances.

\section{SUMMARY AND CONCLUSIONS}

1. Nine patients with Wernicke's syndrome, characterized by ophthalmoplegia, nystagmus, ataxia, and mental disturbances were given a purified diet composed solely of glucose and minerals. Specific vitamins were added after appropriate intervals of observation.

2. Prior to the administration of thiamine, there was no improvement in any of the signs. More specifically, despite alcohol withdrawal, bed rest, and the addition of other vitamins (niacin, calcium pantothenate, pyridoxine, folic acid, ascorbic acid, riboflavin, $\left.\mathrm{B}_{12}\right)$, the ophthalmoplegia progressed and the ataxia remained unchanged, while the nystagmus decreased only in association with an increase in ocular paralysis.

3. When thiamine only was added to the purified diet, the ophthalmoplegia cleared considerably in from one and a quarter to six hours; diminution in nystagmus and ataxia also occurred in some of the patients, but the change was more gradual; there was improvement in mental disturbance, but it was minimal in degree and consisted of increased attentiveness and capacity to maintain a conversation and greater ease of confabulation.

4. In view of these observations, there seems to be little doubt that the ophthalmoplegia of Wernicke's syndrome is related to a specific lack of thiamine. The nystagmus and ataxia also appear to be related to thiamine deficiency, but the evidence is less conclusive. No definite conclusions can be drawn regarding the relationship of the mental disturbances to the deprivation of thiamine or other vitamins.

\section{REFERENCES}

1. Wernicke, C., Lehrbuch der Gehirnkrankheiten fur Aerzte und Studirende. Theodor Fischer, Kassel, 1881, p. 229.

2. Spillane, J. D., Nutritional Disorders of the Nervous System. Williams and Wilkins, Baltimore, 1947 , p. 82.

3. Alexander, L., Wernicke's disease. Identity of lesions produced experimentally by $B_{1}$ avitaminosis in pigeons with hemorrhagic polioencephalitis occurring in chronic alcoholism in man. Am. J. Path., 1940, 16, 61.

4. Riggs, H. E., and Boles, R. S., Wernicke's disease. A clinical and pathological study of 42 cases. Quart. J. Stud. Alcohol, 1944, 5, 361.

5. Campbell, A. C. P., and Biggart, J. H., Wernicke's encephalopathy (polioencephalitis haemorrhagica superior): its alcoholic and non-alcoholic incidence. J. Path. \& Bact., 1939, 48, 245.

6. Campbell, A. C. P., and Russell, W. R., Wernicke's encephalopathy: the clinical features and their probable relationship to vitamin $B$ deficiency. Quart. J. Med., 1941, N.S. 10, 34, 41.

7. de Wardener, H. E., and Lennox, B., Cerebral beriberi (Wernicke's encephalopathy). Review of 52 cases in a Singapore prisoner-of-war hospital. Lancet, 1947, 1, 11.

8. Cruickshank, E. K. Wernicke's encephalopathy. Quart. J. Med., 1950, N.S. 19, 43, 327.

9. Prickett, C. O., The effect of a deficiency of vitamin $B_{1}$ upon the central and peripheral nervous systems of the rat. Am. J. Physiol., 1934, 107, 459.

10. Church, C. F., Functional studies of the nervous system in experimental beriberi. Am. J. Physiol., 1935, 111, 660.

11. Green, R. G., and Evans, C. A., A deficiency disease of foxes. Science, 1940, N.S. 92, 154. 
12. Owen, P. S., and Ferrebee, J. W., The anti-thiamine factor in fish. New England J. Med., 1943, 229, 435.

13. Alexander, L., Pijoan, M., and Myerson, A., Beriberi and scurvy; an experimental study. Tr. Am. Neurol. A., 1938, 64, 135.

14. Swank, R. L., and Prados, M., Avian thiamine deficiency. II. Pathologic changes in the brain and cranial nerves (especially the vestibular) and their relation to the clinical behavior. Arch. Neurol. \& Psychiat., 1942, 47, 97.

15. Jolliffe, N., Wortis, H., and Fein, H. D., The Wernicke syndrome. Arch. Neurol. \& Psychiat., 1941, 46, 569.

16. Wortis, H., Bueding, E., Stein, M. H., and Jolliffe, N., Pyruvic acid studies in the Wernicke syndrome. Arch. Neurol. \& Psychiat., 1942, 47, 215.

17. Victor, M., and Adams, R. D. ( $T_{0}$ be published.)

\section{SPECIAL NOTICE TO SUBSCRIBERS}

Post Offices will no longer forward the Journal when you move.

Please notify The Journal of Clinical Investigation, Business Office, 622 West 168th Street, New York 32, N. Y. at once when you have a change of address, and do not omit the zone number if there is one. 